\title{
Research Article \\ Dynamics and Control on a Discrete Multi-Inventory System
}

\author{
Yuzhen Wang and Li Xu $\mathbb{D}$ \\ School of Science, Tianjin University of Commerce, Tianjin 300134, China \\ Correspondence should be addressed to Li Xu; beifang_xl@163.com
}

Received 12 February 2019; Revised 8 April 2019; Accepted 23 April 2019; Published 12 May 2019

Academic Editor: Michela Robba

Copyright (C) 2019 Yuzhen Wang and Li Xu. This is an open access article distributed under the Creative Commons Attribution License, which permits unrestricted use, distribution, and reproduction in any medium, provided the original work is properly cited.

In this paper, a discrete two item inventory model for deteriorating items with a linear stock dependent demand rate without regular supply is given first; then, regular supply is taken into account, which may be regarded as feedback control. An objective function is formed to calculate the net profit with respect to possible profits and possible loss. A necessary criterion for the steady state optimal control problem for optimizing the objective function subjected to the constraints given by the difference equations of the inventory is obtained. At last, an improved model is put forward.

\section{Introduction}

Inventory management is one of the most important drivers for an effective enterprise. The mathematical modeling of inventory control for inventory management has caught up the mind of inventory researchers. Since the classical economic order quantity model which undoubtedly constitutes the cornerstone of inventory research [1], there are many models in the literature which can illustrate the behavior of inventory management, including not only the continuoustime framework (see [2-5], and the listed conference), but also discrete time one (for example, see $[6,7]$ ), whose origins may be traced in the seminal work of Arrow [8].

In recent years, there are strong calls for the development of multi-item deterioration inventory system management [9]. In general, deterioration is defined as decay, damage, spoilage, evaporation, obsolescence, pilferage, loss of utility, or loss of marginal value of a commodity that results in decrease of usefulness from the original one. Because of deterioration, a certain fraction of the items is either damaged or decayed which will not satisfy the future demand of customers better. Deterioration for such items is continuous and constant or time-dependent. A number of research papers have already been published on the above type of items by some authors, the details of which can be found in the articles [10-17].
For example, some principles which occur in population dynamics can be applied to the multi -item deterioration inventory system in [17]. A two item competitive ordinary differential inventory model for deteriorating items with a linear stock dependent demand rate is given. By means of Pontryagin's Maximum Principle, the author derived a necessary condition for the steady state optimal control problem in which objective function subjected to the constraints given by the competitive ordinary differential equations. The idea is interesting.

In most models, it is assumed that the system events are monitored continuously and that the time axis is continuous. In fact, the inventory systems, in which events occur and decisions are made during fixed length intervals, are characterized, then discrete time inventory models are relatively more reasonable. And, there is a growing research interest in discrete time queues (see [11-14]), which are mainly motivated by their applications in the computer and communication systems. In such a discrete time model, events occur at evenly-spaced points in time $t=0,1,2, \ldots \mathrm{N}$, where the integers represent any unit of time such as a day, a week, or a month. A discrete time system is a more natural manner to describe inventory dynamics.

The model of discrete system control is both a simulation model of inventory dynamics and an optimization model which can give optimal control according to the defined performance criterion [18]. Here, the key idea is to construct 
a function called the Hamiltonian by introducing the adjoint function to attach the difference equation to the objective function, which can be dealt with by the discrete version of Pontryagin's Maximum Principle [19]. For the objective functional subject to the state difference equation with initial condition, the Hamiltonian can be used to find the optimal control. So far, to our knowledge, there have been very few corresponding research works focusing on discrete version of Pontryagin's Maximum Principle on discrete inventory model for deteriorating items. It will be interesting and meaningful.

In this paper, corresponding to [17], a new discrete two item inventory model for deteriorating items with a linear stock dependent demand rate without regular supply is given first, then regular supply is taken into account. So this paper is organized as follows: in Section 2 we give some notations and assumptions. In Section 3, stability analysis is discussed for our discrete two item inventory model for deteriorating items without regular supply and with regular supply. In Section 4, an objective function is formed to calculate the net profit with respect to all possible profits and all possible loss. By means of the discrete version of Pontryagin's Maximum Principle, a necessary condition for the steady state optimal control problem for optimizing the objective function subjected to the constraints given by the difference equations of the inventory is obtained. The next section is simulation discussion; a numerical example is used to illustrate how to find the optimal control and the corresponding state. Then an improved optimal problem with feedback control is put forward. The conclusion will be given in the last section.

\section{Notations and Assumptions}

For convenience, we use the similar assumptions and notation used in [17] which are as follows:

\subsection{Notations}

$i$ : the index of products $i=1,2$

$x_{i}(0)$ : economic order quantity which enters the inventory at time point $n=0, x_{i 0}=x_{i}(0)$

$x_{i}(n)$ : inventory level at discrete time point $n$

$N$ : number of discrete time periods (inventory cycle length)

$k_{i}$ : rate of supply to $x_{i}(n)$ which is inversely proportional to $x_{i}(n)$ and may be $k_{i}=a_{i} / x_{i}(n), a_{i}$ are scale parameter

$d_{i} x_{i}(n)$ : linear demand rate at the instantaneous level of inventory $x_{i}(n)$ at discrete time point $n, d_{i}$ are scale parameter

$a_{i i}$ : deterioration coefficient due to self-contact of $x_{i}(n)$ (self-deterioration coefficient)

$a_{i j}(i \neq j)$ : deterioration coefficient of $x_{i}(n)$ due to presence of unit of $x_{j}(n)$ at discrete time point $n$ (cross-deterioration coefficient)
$K$ : order cost for one order

$c_{i} k_{i}$ : variable cost to maintain supply to $x_{i}(n)$ at the rate $k_{i} x_{i}(n) ; c_{i}$ are scale parameter

$p_{i}$ : price per unit of $x_{i}(n)$

$\theta_{i}$ : the perishable rate of product $x_{i}(n)$

2.2. Assumptions. (1) A product may have a lifetime which ends until its utility reaches zero.

(2) Shortages are not allowed.

(3) The percentage of profit does not depend on the initial inventory.

(4) The rate of changing of holding cost is proportional to the square of $x_{i}$; it is given by $q_{i} x_{i}^{2}, q_{i}$ are constants, $i=1,2$.

\section{Model and Stability Analysis}

In this section, we will show the discrete model which can express the multi-item deterioration inventory. It can also be seen as the discrete version of the model in [17], which can be explained by some principles occurring in population dynamics. Furthermore, stability conditions will be discussed for our discrete two item inventory model without regular supply and with regular supply.

Case 1 (without regular supply). We give the following two item discrete inventory model

$$
\begin{aligned}
& x_{1}(n+1) \\
& \quad=x_{1}(n)\left[1-d_{1}-\theta_{1}-a_{11} x_{1}(n)-a_{12} x_{2}(n)\right] \\
& x_{2}(n+1) \\
& \quad=x_{2}(n)\left[1-d_{2}-\theta_{2}-a_{21} x_{1}(n)-a_{22} x_{2}(n)\right]
\end{aligned}
$$

where $d_{1}, d_{2}, \theta_{1}, \theta_{2}, a_{11}, a_{12}, a_{21}, a_{22}$ are nonnegative, and the above system has four fixed points $(0,0),\left(0,-\left(d_{2}+\theta_{2}\right) / a_{22}\right)$, $\left(-\left(d_{1}+\theta_{1}\right) / a_{11}, 0\right)$ and $\left(\left(-\left(d_{1}+\theta_{1}\right) a_{22}-\left(d_{2}+\theta_{2}\right) a_{12}\right) /\left(a_{11} a_{22}-\right.\right.$ $\left.\left.a_{12} a_{21}\right),\left(-\left(d_{1}+\theta_{1}\right) a_{21}-\left(d_{2}+\theta_{2}\right) a_{11}\right) /\left(a_{11} a_{22}-a_{12} a_{21}\right)\right),(0,0)$ is the only nonnegative one. The linearization of $(1)$ about $(0,0)$ has the Jacobian matrix

$$
J=\left[\begin{array}{cc}
1-d_{1}-\theta_{1} & 0 \\
0 & 1-d_{2}-\theta_{2}
\end{array}\right],
$$

which has two eigenvalues

$$
\lambda_{i}=d_{i}+\theta_{i}-1, \quad i=1,2
$$

if $|\lambda i|<1$ or $d_{i}+\theta_{i}<2$, then steady state $(0,0)$ is locally asymptotically stable; this means that the inventory is empty at last. Then we discuss the case with supply.

Case 2 (with regular supply). If the regular supply $k_{i} x_{i}$ is taken into account, the following model can be given 


$$
\begin{aligned}
x_{1}( & +1) \\
= & x_{1}(n)\left[1-d_{1}-\theta_{1}-a_{11} x_{1}(n)-a_{12} x_{2}(n)\right] \\
& +k_{1} x_{1}(n) \\
x_{2}( & +1) \\
= & x_{2}(n)\left[1-d_{2}-\theta_{2}-a_{21} x_{1}(n)-a_{22} x_{2}(n)\right] \\
& +k_{2} x_{2}(n)
\end{aligned}
$$

Clearly, system (4) has four possible steady states, i.e., $E_{0}=(0,0)$, exclusion points $E_{1}=\left(0,\left(k_{2}-\left(d_{2}+\theta_{2}\right)\right) / a_{22}\right)$, $E_{2}=\left(\left(k_{1}-\left(d_{1}+\theta_{1}\right)\right) / a_{11}, 0\right)$, and nontrivial coexistence point $E_{3}=\left(x_{1}^{*}, x_{2}^{*}\right)$, where

$$
\begin{aligned}
& x_{1}^{*}=\frac{\left[k_{1}-\left(d_{1}+\theta_{1}\right)\right] a_{22}-\left[k_{2}-\left(d_{2}+\theta_{2}\right)\right] a_{12}}{a_{11} a_{22}-a_{12} a_{21}} \\
& x_{2}^{*}=\frac{\left[k_{2}-\left(d_{2}+\theta_{2}\right)\right] a_{11}-\left[k_{1}-\left(d_{1}+\theta_{1}\right)\right] a_{21}}{a_{11} a_{22}-a_{12} a_{21}}
\end{aligned}
$$

and $k_{i}-d_{i}+\theta_{i}>0, i=1,2, a_{11} a_{22}>a_{21} a_{12}, a_{11} / a_{22}>\left(k_{1}-\right.$ $\left.d_{1}-\theta_{1}\right) /\left(k_{2}-d_{2}-\theta_{2}\right)>a_{12} / a_{22}$ or $a_{11} a_{22}<a_{21} a_{12}, a_{11} / a_{22}<$ $\left(k_{1}-d_{1}-\theta_{1}\right) /\left(k_{2}-d_{2}-\theta_{2}\right)<a_{12} / a_{22}$.

At the fixed point $E_{0}$, the Jacobian matrix has the form

$$
J_{E_{1}}=\left[\begin{array}{cc}
k_{1}+1-d_{1}-\theta_{1} & 0 \\
0 & k_{2}+1-d_{2}-\theta_{2}
\end{array}\right] \text {, }
$$

and the corresponding eigenvalues of (6) are

$$
\begin{aligned}
& \lambda_{1}=d_{1}+\theta_{1}-1-k_{1}, \\
& \lambda_{2}=d_{2}+\theta_{2}-1-k_{2} .
\end{aligned}
$$

If $|\lambda i|<1$ or $0<d_{i}+\theta_{i}-k_{i}<2, i=1,2$, then steady state $E_{0}$ is locally asymptotically stable. That is to say, if rate of supply exceeds the sum of the natural deterioration rate and linear demand rate at the instantaneous level of inventory, the inventory will not be empty.

The linearization of (4) about $E_{1}$ has the Jacobian matrix and the corresponding eigenvalues are

$$
\begin{aligned}
& \lambda_{1}=1-d_{1}-\theta_{1}+k_{1}-a_{12} \frac{k_{2}-\left(d_{2}+\theta_{2}\right)}{a_{22}} \\
& \lambda_{2}=1-d_{2}-\theta_{2}+k_{2}-a_{22} \frac{k_{2}-\left(d_{2}+\theta_{2}\right)}{a_{22}} .
\end{aligned}
$$

If $|\lambda i|<1$ or $d_{2}+\theta_{2}<k_{2}<d_{2}+\theta_{2}+1$ and $d_{1}+\theta_{1}+$ $\left(a_{12} / a_{22}\right)\left(k_{2}-d_{2}-\theta_{2}\right)-2<k_{2}<d_{1}+\theta_{1}+\left(a_{12} / a_{22}\right)\left(k_{2}-d_{2}-\right.$ $\left.\theta_{2}\right)$, then steady state $E_{1}$ is locally asymptotically stable. From this, we can conclude that if the above conditions are met, the number of $x_{1}$ will go to zero, and the number of $x_{2}$ will go to $\left(k_{2}-\left(d_{2}+\theta_{2}\right)\right) / a_{22}$.
At the fixed point $E_{2}$, the corresponding stable conditions can be given as follows:

$$
\begin{aligned}
& d_{1}+\theta_{1}<k_{1}<d_{1}+\theta_{1}+1 \\
& d_{12}+\theta_{2}+\frac{a_{21}}{a_{11}}\left(k_{1}-d_{1}-\theta_{1}\right)-2<k_{2} \\
& <d_{12}+\theta_{2}+\frac{a_{21}}{a_{11}}\left(k_{1}-d_{1}-\theta_{1}\right)
\end{aligned}
$$

Similarly, the number of $x_{2}$ will go to zero, and the number of $x_{1}$ will go to $\left(k_{1}-\left(d_{1}+\theta_{1}\right)\right) / a_{11}$.

For the fixed point $E_{3}$, the Jacobian matrix has the form

$$
\begin{aligned}
& J_{E_{3}} \\
& =\left[\begin{array}{cc}
1-\frac{r_{1} a_{11} a_{22}-r_{2} a_{11} a_{12}}{a_{11} a_{22}-a_{12} a_{21}} & -\frac{r_{1} a_{12} a_{22}-r_{2} a_{12}^{2}}{a_{11} a_{22}-a_{12} a_{21}} \\
-\frac{r_{2} a_{11} a_{21}-r_{1} a_{21}^{2}}{a_{11} a_{22}-a_{12} a_{21}} & 1-\frac{r_{2} a_{11} a_{22}-r_{1} a_{21} a_{22}}{a_{11} a_{22}-a_{12} a_{21}}
\end{array}\right] .
\end{aligned}
$$

where $r_{1}=1-d_{1}-\theta_{1}+k_{1}, r_{2}=1-d_{2}-\theta_{2}+k_{2}$.

The characteristic equation of $J_{E_{3}}$ is given by

$$
\lambda^{2}-\operatorname{tr}(J) \lambda+\operatorname{det}(J)=0,
$$

where

$$
\begin{aligned}
& \operatorname{tr}(J)=2-\frac{\left(r_{1}+r_{2}\right) a_{11} a_{22}-r_{2} a_{11} a_{12}-r_{1} a_{21} a_{22}}{a_{11} a_{22}-a_{12} a_{21}} \\
& \operatorname{det}(J)=1+r_{1} r_{2} \\
& \quad-\frac{\left(r_{1}+r_{2}\right) a_{11} a_{22}+\left(r_{2}^{2}-r_{2}\right) a_{11} a_{12}+\left(r_{1}^{2}-r_{1}\right) a_{21} a_{22}}{a_{11} a_{22}-a_{12} a_{21}} .
\end{aligned}
$$

According to the Jury's criteria [20], $E_{3}$ is asymptotically stable if the following conditions hold:

$$
\begin{aligned}
F(-1) & =1-\operatorname{tr}(J) *(-1)+\operatorname{det}(J)>0 \\
F(1) & =1-\operatorname{tr}(J) *(1)+\operatorname{det}(J)>0 \\
1-\operatorname{det}(J) & >0,
\end{aligned}
$$

then we have the following conclusion that if $\left(r_{1} r_{2}+2\right) G>$ $\left(r_{1}+r_{2}\right) a_{11} a_{22}+\left(r_{2}^{2}-r_{2}\right) a_{11} a_{12}+\left(r_{1}^{2}-r_{1}\right) a_{21} a_{22}>r_{1} r_{2} G, G=$ $a_{11} a_{22}-a_{12} a_{21}$ holds, the fixed point $E_{3}$ is asymptotically stable. We can say that $x_{1}$ and $x_{2}$ can coexist.

\section{Necessary Conditions for Dynamic Optimality}

From Case 1 in Section 2, it is a fact that the inventory will be empty at last if $d_{i}+\theta_{i}<2$. On the other hand, it 
is unreasonable if $d_{i}+\theta_{i}$ is too large. The adjustment for the system is necessary which will result in Case 2. From the control theory, the supply action may be regarded as a feedback control one. In other words, one may wish to move the undesired trajectory towards the desired orbit. Here we will alter the positions of positive equilibrium, but to obtain its stability. This motivates us to adjust the model form as follows.

$$
\begin{aligned}
& x_{1}(n+1)=x_{1}(n) \\
& \cdot {\left[1-d_{1}-\theta_{1}-a_{11} x_{1}(n)-a_{12} x_{2}(n)\right]+k_{1} x_{1}(n) } \\
& \quad x_{1}(n) \\
& \cdot {\left[1-d_{1}-\theta_{1}-a_{11} x_{1}(n)-a_{12} x_{2}(n)+b_{1} u_{1}(n)\right] } \\
& x_{2}(n+1)=x_{2}(n) \\
& \cdot\left[1-d_{2}-\theta_{2}-a_{21} x_{1}(n)-a_{22} x_{2}(n)\right]+k_{2} x_{2}(n) \\
& \quad=x_{2}(n) \\
& \cdot\left[1-d_{2}-\theta_{2}-a_{21} x_{1}(n)-a_{22} x_{2}(n)+b_{2} u_{2}(n)\right]
\end{aligned}
$$

where $k_{1}=b_{1} u_{1}(n)$ and $k_{2}=b_{2} u_{2}(n), u_{1}$ and $u_{2}$ are control variables.

Under the condition the inventory will not be empty; we will calculate the net profit of the inventory. The objective function for the inventory problem should first be formed. Note that the percentage of profit does not depend on the initial inventory and the nonnegativity constraints hold for inventory variables, and percentage of profit maybe is found to be more for a lower initial stock than that for a higher initial stock [17]. Naturally we can formulate the optimization problem to maximize the net profit (performing index) $G$ taking the form

$$
\begin{aligned}
G= & -K+\sum_{i=1}^{2} \sum_{n=0}^{N-1}\left[p_{i} d_{i} x_{i}(n)-\left(p_{i} a_{i i}+q_{i}\right) x_{i}^{2}(n)\right] \\
& +\sum_{i=1}^{2} \sum_{n=0}^{N-1}\left[p_{i}-\frac{c_{i} b_{i} u_{i}(n)}{x_{i}(n)}\right] x_{i}(n) \\
& \cdot\left[-d_{i}-\theta_{i}-a_{i i} x_{i}(n)-a_{i j} x_{j}(n)+b_{i} u_{i}(n)\right]
\end{aligned}
$$

$$
\begin{aligned}
& \text { s.t } \\
& \begin{array}{l}
x_{1}(n+1)=x_{1}(n) \\
\cdot\left[1-d_{1}-\theta_{1}-a_{11} x_{1}(n)-a_{12} x_{2}(n)+b_{1} u_{1}(n)\right] \\
x_{2}(n+1)=x_{2}(n) \\
\cdot\left[1-d_{2}-\theta_{2}-a_{21} x_{1}(n)-a_{22} x_{2}(n)+b_{2} u_{2}(n)\right] .
\end{array}
\end{aligned}
$$

and

$$
\begin{gathered}
\left(r_{1} r_{2}+2\right) G> \\
\left(r_{1}+r_{2}\right) a_{11} a_{22}+\left(r_{2}^{2}-r_{2}\right) a_{11} a_{12} \\
+\left(r_{1}^{2}-r_{1}\right) a_{21} a_{22}>r_{1} r_{2} G,
\end{gathered}
$$

where $G=a_{11} a_{22}-a_{12} a_{21}, r_{1}=1-d_{1}-\theta_{1}+b_{1} u_{1}, r_{2}=$ $1-d_{2}-\theta_{2}+b_{2} u_{2}$.

For simplicity, we note $S=\left\{\left(d_{1}, d_{2}, \theta_{1}, \theta_{2}, b_{1}, b_{2}, a_{11}, a_{12}\right.\right.$, $\left.a_{21}, a_{22}\right) \mid\left(r_{1} r_{2}+2\right) G>\left(r_{1}+r_{2}\right) a_{11} a_{22}+\left(r_{2}^{2}-r_{2}\right) a_{11} a_{12}+\left(r_{1}^{2}-\right.$ $\left.\left.r_{1}\right) a_{21} a_{22}>r_{1} r_{2} G\right\}$.

We can easily obtain the next results.

Theorem 1. There exists an optimal control $u^{*}=\left(u_{1}^{*}, u_{2}^{*}\right)$ with corresponding states from (14) such that $G\left(u^{*}\right)=\min G(u)$.

Now we consider the Hamiltonian

$$
\begin{aligned}
& H(n)=-\frac{K}{N}+\sum_{i=1}^{2}\left(p_{i} d_{i} x_{i}(n)-\left(p_{i} a_{i i}+q_{i}\right) x_{i}(n)^{2}\right) \\
& -\sum_{i=1}^{2}\left[p_{i}-\frac{c_{i} b_{i} u_{i}(n)}{x_{i}(n)}\right] x_{i}(n) \\
& \cdot\left[1-d_{i}-\theta_{i}-a_{i i} x_{i}(n)-a_{i j} x_{j}(n)+b_{i} u_{i}(n)\right] \\
& +\lambda_{1}(n+1) x_{1}(n) \\
& \cdot\left[1-\left(d_{1}+\theta_{1}\right)-a_{11} x_{1}(n)-a_{12} x_{2}(n)+b_{1} u_{1}(n)\right] \\
& +\lambda_{2}(n+1) x_{2}(n) \\
& \cdot\left[1-\left(d_{1}+\theta_{1}\right)-a_{21} x_{1}(n)-a_{22} x_{2}(n)+b_{2} u_{2}(n)\right],
\end{aligned}
$$

$\lambda_{i}$ are the costate variables, then, from discrete Pontryagin's Maximum Principle [19], we can derive the necessary conditions in the next theorem.

Theorem 2. Given an optimal control $u^{*}=\left(u_{1}^{*}, u_{2}^{*}\right)$ and corresponding states $x_{1}^{*}$ and $x_{2}^{*}$ from (14), there exist adjoint functions $\lambda_{1}$ and $\lambda_{2}$ satisfying

$$
\begin{aligned}
& \lambda_{1}(n)=p_{1} d_{1}-2\left(p_{1} a_{11}+q_{1}\right) x_{1}(n) \\
& +\left(p_{1} x_{1}-c_{1} b_{1} u_{1}\right) a_{11}-p_{1} x_{1}(n+1)+\lambda_{1}(n+1) \\
& \cdot\left[1-\left(d_{1}+\theta_{1}\right)-2 a_{11} x_{1}(n)-a_{12} x_{2}(n)\right] \\
& \lambda_{2}(n)=p_{2} d_{2}-2\left(p_{2} a_{22}+q_{2}\right) x_{2}(n) \\
& +\left(p_{2} x_{2}-c_{2} b_{2} u_{2}\right) a_{22}-p_{2} x_{2}(n+1)+\lambda_{2}(n+1) \\
& \cdot\left[1-\left(d_{2}+\theta_{2}\right)-a_{21} x_{1}(n)-2 a_{22} x_{2}(n)\right] \\
& \lambda_{1}(N)=\lambda_{2}(N)=0,
\end{aligned}
$$


and

$$
\lambda_{i}(n+1)=\frac{p_{i} x_{i}(n)-c_{i} b_{i} u_{i}(n)-c_{i} x_{i}(n+1)}{x_{i}(n)}
$$

at $u_{i}^{*}(n)$.

where $n=0,1,2, \ldots, N-1$.

Proof. Using the discrete version of Pontryagin's Maximum Principle

$$
\begin{aligned}
& \lambda_{1}(n)=\frac{\partial H(n)}{\partial x_{1}(n)}=p_{1} d_{1}-2\left(p_{1} a_{11}+q_{1}\right) x_{1}(n) \\
& +\left(p_{1} x_{1}-c_{1} b_{1} u_{1}\right) a_{11}-p_{1} x_{1}(n+1)+\lambda_{1}(n+1) \\
& \cdot\left[1-\left(d_{1}+\theta_{1}\right)-2 a_{11} x_{1}(n)-a_{12} x_{2}(n)\right] \\
& \lambda_{2}(n)=\frac{\partial H(n)}{\partial x_{2}(n)}=p_{2} d_{2}-2\left(p_{2} a_{22}+q_{2}\right) x_{2}(n) \\
& +\left(p_{2} x_{2}-c_{2} b_{2} u_{2}\right) a_{22}-p_{2} x_{2}(n+1)+\lambda_{2}(n+1) \\
& \cdot\left[1-\left(d_{2}+\theta_{2}\right)-a_{21} x_{1}(n)-2 a_{22} x_{2}(n)\right],
\end{aligned}
$$

Additionally, from the adjoint final conditions, we can get

$$
\lambda_{1}(N)=\lambda_{2}(N)=0
$$

Furthermore,

$$
\begin{aligned}
& \frac{\partial H(n)}{\partial u_{i}} \\
& =c_{i}\left[1-d_{i}-\theta_{i}-a_{i i} x_{i}(n)-a_{i j} x_{j}(n)+b_{i} u_{i}(n)\right] \\
& \quad-\left(p_{i} x_{i}(n)-c_{i} b_{i} u_{i}\right)+\lambda_{i}(n+1) x_{i} \\
& =c_{i} x_{i}(n+1)-\left(p_{i} x_{i}(n)-c_{i} b_{i} u_{i}(n)\right) \\
& \quad+\lambda_{i}(n+1) x_{i}(n)=0, \quad \text { at } u_{i}^{*}(n)
\end{aligned}
$$

$x_{i} \neq 0$,

from which we can get $\lambda_{i}(n+1)=\left(p_{i} x_{i}(n)-c_{i} b_{i} u_{i}(n)-c_{i} x_{i}(n+\right.$ $1)) / x_{i}(n)$.

\section{Simulation and Discussion}

In this section, we will show a simple example in order to intuitively illustrate how to find the optimal control firstly. We fix some of the constants in the parameter region $S$, and let $d_{1}=0.4, d_{2}=0.3, p_{1}=1, p_{2}=2, \theta_{1}=0.0002, \theta_{2}=$ $0.0003, b_{1}=1, b_{2}=1, a_{11}=0.001, a_{12}=0.02, a_{21}=$ $0.03, a_{22}=0.002$. The initial values will be set to $x_{10}=$ $10, x_{20}=15$, and let $N=10$, by means of forward-backward sweep, the optimal solutions and optimal control can be displayed in Figures 1 and 2.

According to the theoretical analysis in Sections 3 and 4, stability analysis at the steady state of the discrete two item
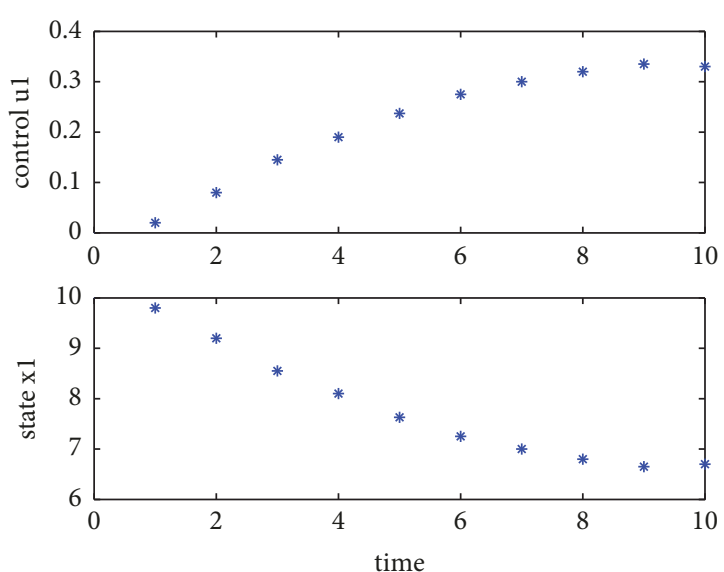

FIgURE 1: Optimal control and state for $x_{1}$ and $u_{1}$ with 10 time steps.
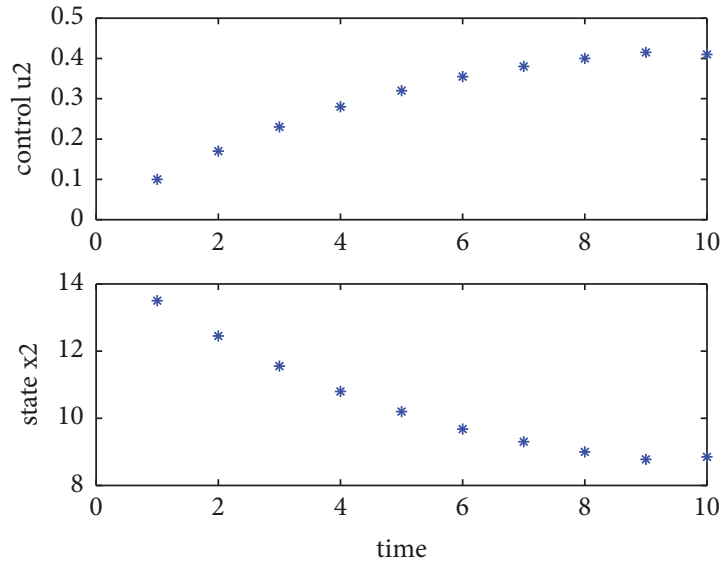

FIGURE 2: Optimal control and state for $x_{2}$ and $u_{2}$ with 10 time steps.

inventory model for deteriorating items with regular supply is done to ensure the warehouse is not empty and keep at an equilibrium level. With the view of control theory, the supply action can also be regarded as a feedback control. And we have altered the positions of positive equilibrium to obtain its stability. In fact, we can rewrite the discrete two item inventory model, in which the control variables satisfy a certain difference equation [21].

$$
\begin{aligned}
& x_{1}(n+1)=x_{1}(n) \\
& \cdot\left[1-d_{1}-\theta_{1}-a_{11} x_{1}(n)-a_{12} x_{2}(n)+b_{1} u_{1}(n)\right] \\
& x_{2}(n+1)=x_{2}(n) \\
& \quad \cdot\left[1-d_{2}-\theta_{2}-a_{21} x_{1}(n)-a_{22} x_{2}(n)+b_{2} u_{2}(n)\right] \\
& u_{1}(n+1)=\left(1-e_{1}\right) u_{1}(n)+d_{1} x_{1}(n) \\
& u_{2}(n+1)=\left(1-e_{2}\right) u_{2}(n)+d_{2} x_{2}(n) .
\end{aligned}
$$

where the parameters $d_{1}, d_{2}, e_{1}$, and $e_{2}$ are positive constants. 
Similarly, an objective function is formed to calculate the net profit with respect to all possible profits and all possible loss for the system with the above state equation. We can obtain an optimal control problem as follows:

$$
\max G(x, u)
$$

s.t

$$
\begin{aligned}
& x_{1}(n+1)=x_{1}(n) \\
& \quad \cdot\left[1-d_{1}-\theta_{1}-a_{11} x_{1}(n)-a_{12} x_{2}(n)+b_{1} u_{1}(n)\right] \\
& x_{2}(n+1)=x_{2}(n) \\
& \quad \cdot\left[1-d_{2}-\theta_{2}-a_{21} x_{1}(n)-a_{22} x_{2}(n)+b_{2} u_{2}(n)\right] \\
& u_{1}(n+1)=\left(1-e_{1}\right) u_{1}(n)+d_{1} x_{1}(n) \\
& u_{2}(n+1)=\left(1-e_{2}\right) u_{2}(n)+d_{2} x_{2}(n) .
\end{aligned}
$$

Corresponding to system (15)-(16), applying the discrete version of Pontryagin's Maximum Principle, a necessary criterion for the steady state optimal control problem for optimizing the objective function subjected to the constraints given by the difference equations (24) may be different. Perhaps the problem is worth discussing.

\section{Conclusion}

In this paper, we first get a new discrete two item inventory model for deteriorating items with a linear stock dependent demand rate without regular supply, but steady state $(0,0)$ is locally asymptotically stable which means that the inventory is empty at last, so regular supply is the fact. To maximize net profits, an objective function is formed with the difference equation constraints with feedback control. Then there is a necessary condition for the steady state optimal control problem by the discrete Pontryagin's Maximum Principle; the numerical can also verify the validity of theoretical analysis. At last, a revised optimal control problem is put forward.

\section{Data Availability}

The data used to support the findings of this study are available from the corresponding author upon request.

\section{Conflicts of Interest}

The authors declare that there are no conflicts of interest regarding the publication of this paper.

\section{Acknowledgments}

This work was supported by Tianjin Municipal Education Commission Scientific Research Project of China (Grant No. 161082) and Applied Study Program (Grant No. 60204 and WH18012).

\section{References}

[1] F. W. Harris, "How many parts to make at once," Operations Research, vol. 10, pp. 135-136, 1913.

[2] E. Naddor, Inventory Systems, Wiley, New York, NY, USA, 1996.

[3] B. M. Worell and M. A. Hall, "The analysis of inventory control model using polynomial geometric programming," International Journal of Production Research, vol. 20, pp. 657667, 1982.

[4] P. H. Zipkin, Foundations of inventory management, McGrawHill, New York, NY, USA, 2000.

[5] A. G. Lagodimos, I. T. Christou, and K. Skouri, "Computing globally optimal (s,S,T) inventory policies," Omega, vol. 40, no. 5, pp. 660-671, 2012.

[6] A. G. Lagodimos, K. Skouri, and I. T. Christou, "Global optimization of two-echelon serial chains with replenishment costs," IMA Journal of Management Mathematics, vol. 28, no. 2, pp. 205-233, 2017.

[7] A. G. Lagodimos, K. Skouri, I. T. Christou, and P. T. Chountalas, "The discrete-time EOQ model: solution and implications," European Journal of Operational Research, vol. 266, no. 1, pp. 112-121, 2018.

[8] K. A. Arrow, T. E. Harris, and J. Marshak, "Optimal inventory policy," Econometrica, vol. 19, no. 3, pp. 250-272, 1951.

[9] E. Nador, Inventory System, John Wiley, New York, NY, USA, 1996.

[10] Z. T. Balkhi, "An optimal solution of a general lot inventory model with deteriorated and imperfect products, taking into account inflation and time value of money," International Journal of System Science, vol. 35, pp. 87-96, 2004.

[11] K. Maity and M. Maiti, "Inventory of deteriorating complementary and substitute items with stock dependent demand," American Journal of Mathematical and Management Sciences, vol. 25, no. 1-2, pp. 83-96, 2005.

[12] Y. W. Zhou, H. S. Lau, and S. L. Yang, "A new variable production scheduling strategy for deteriorating items with time-varying demand and partial lost sale," Computers and Operations research, vol. 3, pp. 1753-1776, 2003.

[13] A. K. Maity, K. Maity, S. Mondal, and M. Maiti, "A Chebyshev approximation for solving the optimal production inventory problem of deteriorating multi-item," Mathematical and Computer Modelling, vol. 45, no. 1-2, pp. 149-161, 2007.

[14] S. K. Goyal and B. C. Giri, "Recent trends in modeling of deteriorating inventory," European Journal of Operational Research, vol. 134, no. 1, pp. 1-16, 2001.

[15] M. Bakker, J. Riezebos, and R. H. Teunter, "Review of inventory systems with deterioration since 2001," European Journal of Operational Research, vol. 221, no. 2, pp. 275-284, 2012.

[16] G. Padmanabhan and P. Vrat, "Analysis of multi-system under resource constraint: a nonlinear goal programming approach," Engineering Construction and Production Management, vol. 13, no. 11, pp. 104-112, 1990.

[17] D. K. Bhattacharya, "On multi-item inventory," European Journal of Operational Research, vol. 162, no. 3, pp. 786-791, 2005.

[18] K. Kostic, "Inventory control as a discrete system control for the fixed-order quantity system," Applied Mathematical Modelling, vol. 33, no. 11, pp. 4201-4214, 2009.

[19] S. M. Lenhart and J. T. Workman, Optimal Control Applied to Biological Models, Chapmal Hall/CRC, Boca Raton, Fla, USA, 2007. 
[20] X. Liu and D. Xiao, "Complex dynamic behaviors of a discrete-time predator-prey system," Chaos, Solitons \& Fractals, vol. 32, no. 1, pp. 80-94, 2007.

[21] F. D. Chen, "Permanence in a discrete Lotka-Volterra competition model with deviating arguments," Nonlinear Analysis: Real World Applications, vol. 9, no. 5, pp. 2150-2155, 2008. 


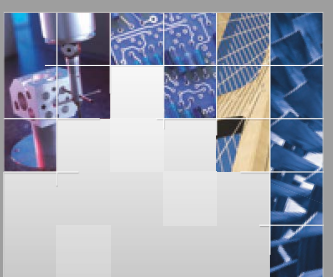

\section{Enfincering}
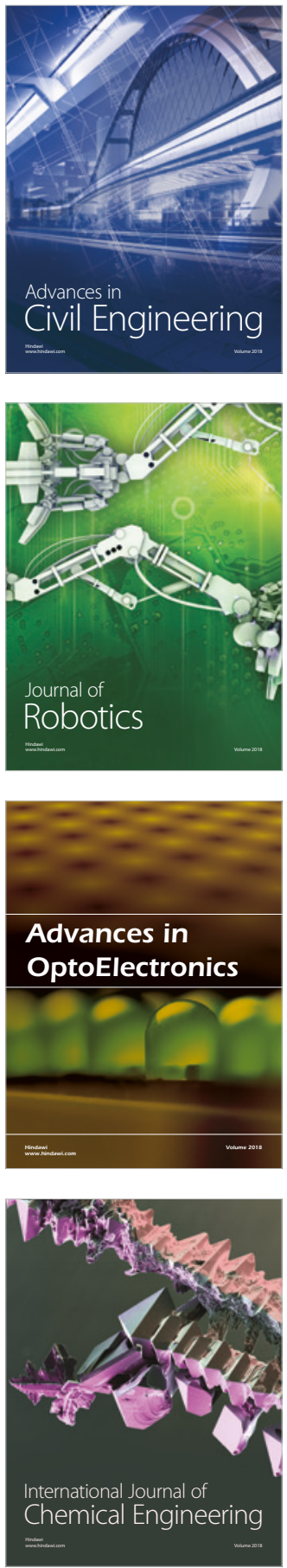

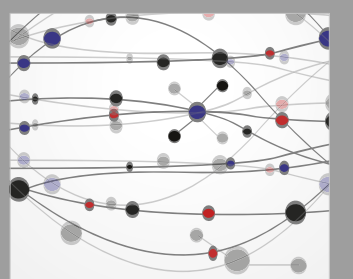

\section{Rotating \\ Machinery}

The Scientific World Journal

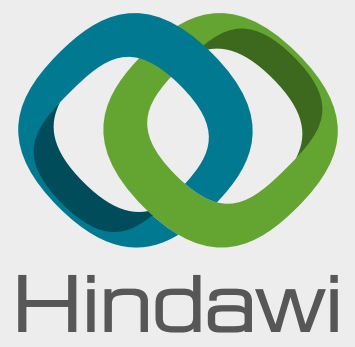

Submit your manuscripts at

www.hindawi.com
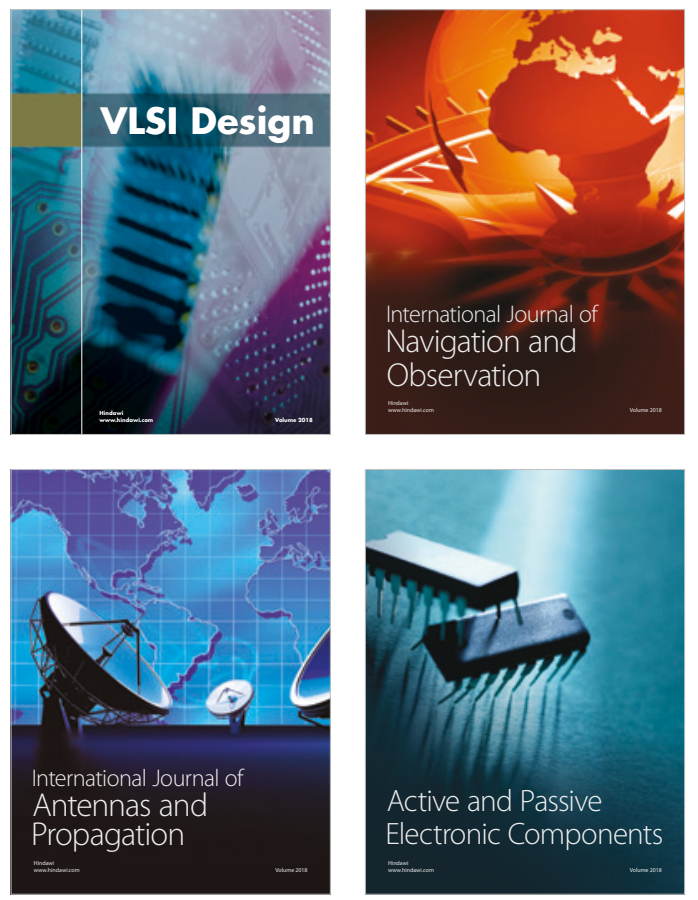
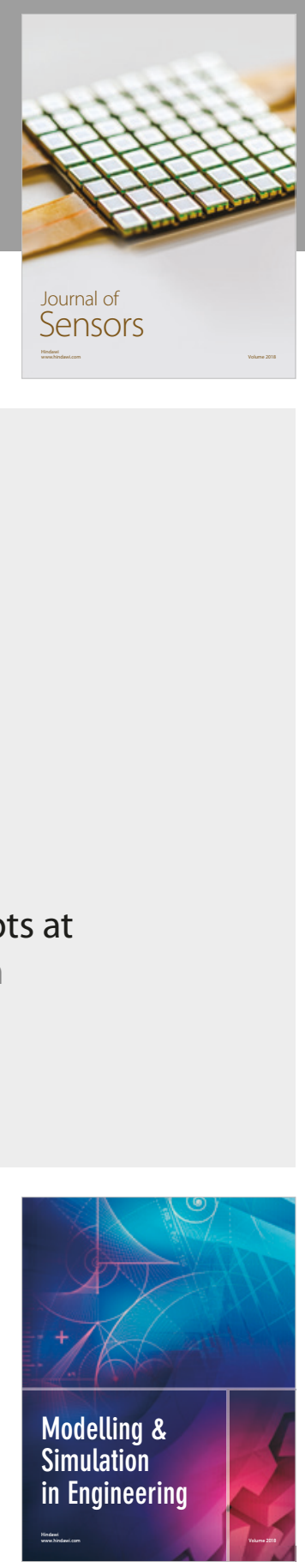

\section{Advances \\ Multimedia}
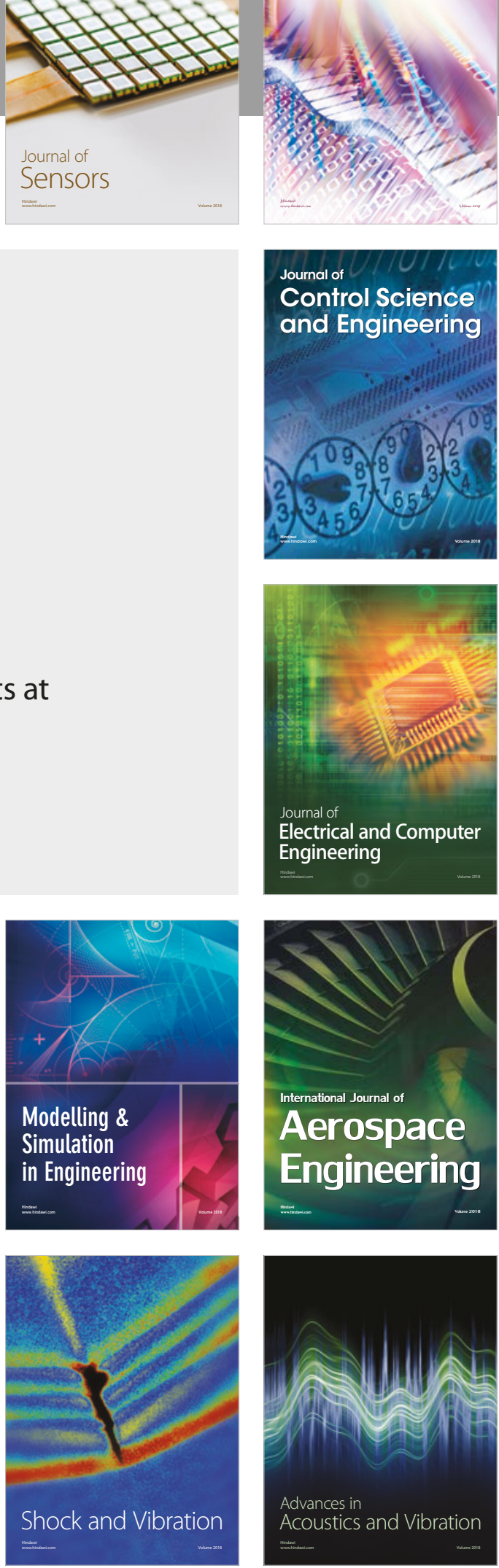